\title{
Identification and Antimicrobial Susceptibility testing of Non Fermenting Gram Negative Bacteria by Vitek 2 in a Teaching Hospital in Mahbubnagar
}

\author{
Srividya Yeruva* and Vasantha Kabra
}

Department of Microbiology, SVS Medical College, Yenugonda, Mahbubnagar, Telangana, 509001, India

*Corresponding author

\begin{tabular}{l} 
K e y w o r d s \\
$\begin{array}{l}\text { NFGNB, Pseudomonas } \\
\text { aeruginosa, Acinetobacter } \\
\text { baumanii, } \\
\text { Stenotrophomonas } \\
\text { maltophilia, Burkholderia } \\
\text { cepacia, Vitek 2, MDR, } \\
\text { PDR }\end{array}$ \\
\hline Article Info \\
$\begin{array}{l}\text { Accepted: } \\
\text { xx September } 2018 \\
\text { Available Online: } \\
\text { xx October } 2018\end{array}$ \\
\hline
\end{tabular}

Non fermenting gram negative bacilli (NFGNB) are aerobic, non-spore forming bacteria. Although previously considered as contaminants, their pathogenic potential was proved by their frequent isolation from clinical samples and association with diseases. Aim: To identify NFGNB from various clinical samples and perform antimicrobial susceptibility testing (AST) by Vitek 2. Materials and methods: All the clinical samples received for routine bacterial culture in Department of Microbiology, SVS Medical College, from January 2017 to April 2018 were plated on agar plates and non-lactose fermenting colonies on MacConkey agar plates were further processed by Vitek 2. Results: A total of 126 NFGNB were isolated from various clinical samples and their AST was evaluated by Vitek 2. Pseudomonas aeruginosa $(66.66 \%)$ and Acinetobacter baumanii $(23.80 \%)$ were most common NFGNB isolated. High variability in antibiotic resistance pattern was noted in the present study. $47.61 \%$ of NFGNB exhibited Multidrug resistance (MDR) and 3.37\% Pseudomonas aeruginosa strains showed Pandrug resistance (PDR). Conclusion: Accurate and rapid identification and antimicrobial susceptibility testing of NFGNB by Vitek 2, help in early initiation of appropriate antimicrobial therapy and proper management of patients and will reduce emergence of MDR and PDR strains of NFGNB.

\section{Introduction}

Non fermenting gram negative bacteria are saprophytic in nature and were previously considered as contaminants or commensals of little significance (Prudhivi Sumana et al., 2017). However their pathogenic potential has been proved beyond doubt by their frequent isolation from clinical material and their association with disease (Susmitha Simgamsetty et al., 2016). Recent literature review also shows that they have emerged as important nosocomial pathogens causing life threatening infections particularly in hospitalised patients, immunocompromised hosts and patients with hematological malignancies. NFGNB are intrinsically resistant to many antibiotics and are known to produce ESBL's and metallo- $\beta$-lactamases. NFGNB consist of hetrogenous group of organisms like Pseudomonas spp, Acinetobacter spp, Alkaligenes spp, Stenotrophomonas maltophilia, Burkholderia cepacia (BCC). Currently Pseudomonas aeruginosa, Acinetobacter baumannii are the most commonly isolated non-fermenters 
pathogenic for humans. Infections caused by other species are relatively infrequent (Prudhivi Sumana et al., 2017).

In recent years due to liberal and empirical use of antibiotics, most of these organisms are now resistant to many routinely used antibiotics causing treatment failures. The emergence of resistance as a major problem has drawn attention to a need for better diagnostic techniques (Ridhima Wadhwa et al., 2016). Vitek 2 is one such instrument which is fully automated system, designed to decrease the turnaround time for the identification of bacteria and determination of antimicrobial susceptibilities. The instrument also provides a more hands-off approach than the original Vitek instrument (Caroline $\mathrm{M}$. O'Hara et al., 2003). The purpose of present study is to identify non-fermenting gram negative bacteria from various clinical samples isolated in a teaching hospital and to evaluate their antimicrobial susceptibility patterns by Vitek 2 method.

\section{Materials and Methods}

The present study was conducted in the Department of Microbiology, SVS medical college, Mahbubnagar during the period from January 2017 to April 2018. All the clinical samples received for bacterial culture were plated on blood agar and MacConkey agar and incubated at $37^{\circ} \mathrm{C}$ for $48 \mathrm{hrs}$. The isolates that showed non lactose fermenting colonies on MacConkey agar were provisionally considered as NFGNB. Further identification and antimicrobial susceptibility testing was performed by subjecting them to Vitek 2 compact system (Prudhivi Sumana et al., 2017).

\section{Inoculum preparation}

From the isolated colonies grown on the media, a bacterial suspension was prepared in
$3 \mathrm{ml}$ of sterile saline in a clear plastic (polystyrene) test tube. The turbidity of the suspension was adjusted to a McFarland standard of 0.5 with the help of a Vitek 2 DensiCheck instrument. The time between the preparation of inoculum and filling of the card was always less than 30 minutes. Identification with the Vitek 2 compact system was performed according to the manufacturer's instructions using the 64 well plastic Gram Negative (GN) card. The culture suspension was inoculated into the GN card with the help of a vacuum device inside the filling chamber. The cards were later transferred into the loading chamber where the cards were sealed and were incubated in a rotating carousel at $37{ }^{\circ} \mathrm{C}$. Each loaded card was removed from the carousel and transported to the optical system for reaction readings and data was collected (Prudhivi Sumana et al., 2017).

\section{Quality control}

The Vitek 2 compact machine was validated using the standard strain as per the manufacturer's instructions. Pseudomonas aeruginosa 27853 was used. During the study period, the control strain was checked at regular intervals.

\section{Antimicrobial susceptibility testing}

Antimicrobial susceptibility testing with the Vitek 2 compact system was performed using an AST N281 card according to the Manufacturer's instructions. A panel of seventeen antibiotics in AST N281 card was tested. The cards were filled with inoculum (Prepared by transferring $200 \mu \mathrm{l}$ of culture suspension from the $0.5 \mathrm{McFarland}$ culture suspension used for filling the identification cards into a fresh $3 \mathrm{ml}$ sterile saline solution obtaining a final turbidity of $8 \times 106 \mathrm{cfu} / \mathrm{ml}$ ) in the filling chamber. The Vitek 2 system automatically processes the antimicrobial 
susceptibility cards until MIC's are obtained. The Vitek 2 compact system subsequently corrects, where necessary for MIC's in accordance with the internal database of possible phenotypes for microorganism antimicrobial agent combinations (Prudhivi Sumana et al., 2017).

\section{Results and Discussion}

A total of 126 non-fermenting gram negative bacteria were isolated from various clinical samples processed by Vitek 2 from January 2017 to April 2018 in SVS Medical College. Majority of NFGNB were isolated from pus/wound swabs $(40.47 \%)$ followed by urine $(21.42 \%)$, blood $(19.04 \%)$, sputum $(6.34 \%)$, body fluids $(4.76 \%)$, bronchioalveolar lavage fluid $(4.76 \%)$ and endotracheal tube tips $(3.17 \%)$.

Among 126 NFGNB isolated in present study, $69.04 \%$ (87) were from patients above 50 years age group, $16.66 \%$ (21) from 20-55 years age group patients and $14.28 \%$ (18) from below 25 years age group patients.

NFGNB that were previously considered to be contaminants have emerged as important nosocomial pathogens. Pseudomonas aeruginosa and Acinetobacter species are known to be common among them (Susmitha Simgamshetty et al., 2016). The Vitek 2 combines several advantages that may be of clinical interest for routine testing of gramnegative rods isolated from clinical samples: rapid identification, simple methodology, a high level of automation and taxonomically updated databases (Providencia Joyanes et al., 2001).

The Vitek 2 compact system identifies NFGNB along with their antibiotic susceptibility pattern with in a time period of 8 to 16hrs (Susmitha Simgamsetty et al., 2016). Earlier the identification of non-fermenters based on biochemical tests was cumbersome and many non-fermenters were misidentified. But now with the availability of commercial system like Vitek 2, the identification has become easier (Prudhivi Sumana et al., 2017).

In the present study majority of NFGNB were isolated from Pus/wound swabs (40.47\%) followed by urine, blood, sputum which is similar to (Shilpa K. Gokale et al., 2012) study and in contrary (Prudhivi Sumana et al., 2017) study majority of NFGNB are isolated from blood and respiratory samples.

Pseudomonas aeruginosa (66.66\%) was commonest NFGNB isolated in present study which is in accordance with (A Malini et al., 2009) and (Susmitha Simgamshetty et al., 2016) studies. A very high percentage of Pseudomonas aeruginosa $(82.3 \%)$ are isolated in (Shilpa K. Gokale et al., 2012) study. In contrast to present study, Acinetobacter baumanii $(46.15 \%)$ is the commonest NFGNB isolated followed by Pseudomonas aeruginosa $(12.62 \%)$ in (Prudhivi Sumana et al., 2017) study (Table 1-6).

Table.1 Distribution of isolated NFGNB Species in present study $(\mathrm{n}=126)$

\begin{tabular}{|c|c|c|}
\hline NFGNB & NO.OF ISOLATES & $\%$ ISOLATES \\
\hline Pseudomonas aeruginosa & 84 & $66.66 \%$ \\
\hline Acinetobacter baumanii & 30 & $23.80 \%$ \\
\hline Burkholderia cepacia & 6 & $4.76 \%$ \\
\hline Stenotrophomonas maltophilia & 6 & $4.76 \%$ \\
\hline
\end{tabular}


Table.2 Comparison of commonest NFGNB isolates in different studies
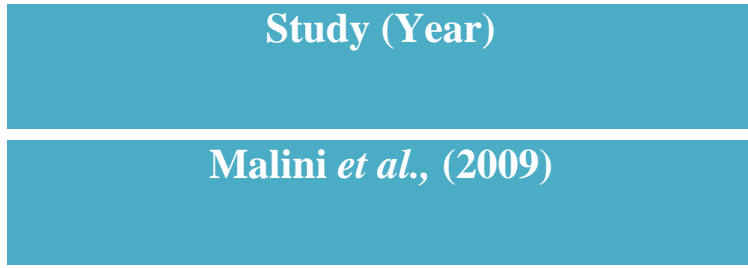

Shilpa K. Gokale et al., (2012)

Susmitha Simgamshetty et al., (2016)

Present study

\begin{tabular}{|c|c|}
\hline $\begin{array}{c}\text { Pseudomonas } \\
\text { aeruginosa }(\%)\end{array}$ & $\begin{array}{c}\text { Acinetobacter } \\
\text { baumanii }(\%)\end{array}$ \\
\hline $53.8 \%$ & $22.2 \%$ \\
\hline $82.3 \%$ & $15.4 \%$ \\
\hline $56.45 \%$ & $37.09 \%$ \\
\hline & \\
\hline $66.66 \%$ & $\mathbf{2 3 . 8 0 \%}$ \\
\hline
\end{tabular}

Table.3 Distribution of NFGNB isolated from samples sent from various areas in hospital

\begin{tabular}{c} 
NFGNB \\
(NO. OF ISOLATES) \\
\hline Pseudomonas aeruginosa (84) \\
\hline Acinetobacter baumanii (30) \\
\hline Burkholderia cepacia (6) \\
\hline Stenotrophomonas maltophilia (6) \\
\hline TOTAL NFGNB (126)
\end{tabular}

\begin{tabular}{|c|c|c|c|}
\hline \% (no.) of NFGNB isolated from various areas in hospital \\
\hline \begin{tabular}{c|c|c|} 
Wards \\
ICUs
\end{tabular} & ER & OPD \\
\hline $10 \%(3)$ & $64.28 \%(54)$ & $3.57 \%(3)$ & $3.57 \%(3)$ \\
\hline $0 \%$ & $80 \%(24)$ & $10 \%(3)$ & $0 \%$ \\
\hline $50 \%(3)$ & $50 \%(3)$ & $0 \%$ & $0 \%$ \\
\hline $\mathbf{2 3 . 8 0 \% ( 3 0 )}$ & $\mathbf{6 9 . 0 4 \% ( 8 7 )}$ & $\mathbf{4 . 7 \% ( 6 )}$ & $\mathbf{2 . 3 8 \% ( 3 )}$ \\
\hline
\end{tabular}

Table.4 Sex-wise distribution of NFGNB

\begin{tabular}{|c|}
\hline Study (Year) \\
\hline Ridhima wadhwa et al., (2016) \\
\hline Kritilaxmi et al., (2018) \\
\hline Present study \\
\hline
\end{tabular}

\begin{tabular}{|c|c|}
\hline Male \% & Female \% \\
\hline $70 \%$ & $30 \%$ \\
\hline $68 \%$ & $32 \%$ \\
\hline $\mathbf{6 9 . 0 4} \%$ & $\mathbf{3 0 . 9 6 \%}$ \\
\hline
\end{tabular}


Table.5 Percentage (\%) of NFGNB sensitive to antibiotics tested by Vitek 2 AST Panel

\begin{tabular}{|c|}
\hline $\begin{array}{c}\text { ANTIBIOTIC } \\
\text { (code) }\end{array}$ \\
\hline Amikacin (AN) \\
\hline Aztreonam (ATM) \\
\hline Cefepime (FEP) \\
\hline Cefperazone/Sulbactum (SFP) \\
\hline Ceftaridime (CAZ) \\
\hline Ciprofloxacin (CIP) \\
\hline Colistin (CS) \\
\hline Doripenem (DOR) \\
\hline Gentamicin (GM) \\
\hline Imipenem (IPM) \\
\hline Levofloxacin (LEV) \\
\hline Meropenem (MIEM) \\
\hline Minocyclin (MNO) \\
\hline Piperacillin/Tazobactam (TZP) \\
\hline Ticarcillin/Clavulanic Acid (TCC) \\
\hline Tigecycline (TGC) \\
\hline Trimethoprim/Sulfamethoxazole(SXT)
\end{tabular}

\begin{tabular}{|c|c|c|c|}
\hline \multicolumn{4}{|c}{ Antibiotic Sensitivity Percentage (\%) of NFGNB } \\
\hline $\begin{array}{c}\text { Pseudomonas } \\
\text { aeruginosa (84) }\end{array}$ & $\begin{array}{c}\text { Acinetobacter } \\
\text { baumanii (30) }\end{array}$ & $\begin{array}{c}\text { Burkholderia } \\
\text { cepacia (6) }\end{array}$ & $\begin{array}{c}\text { Stenotrophomonas } \\
\text { maltophilia (6) }\end{array}$ \\
\hline $42.85 \%$ & $0 \%$ & $0 \%$ & $0 \%$ \\
\hline $32.14 \%$ & $0 \%$ & $0 \%$ & $0 \%$ \\
\hline $50 \%$ & $10 \%$ & $0 \%$ & $0 \%$ \\
\hline $50 \%$ & $10 \%$ & $50 \%$ & $0 \%$ \\
\hline $39.28 \%$ & $0 \%$ & $0 \%$ & $0 \%$ \\
\hline $25 \%$ & $0 \%$ & $0 \%$ & $100 \%$ \\
\hline $96.42 \%$ & $100 \%$ & $0 \%$ & $100 \%$ \\
\hline $53.57 \%$ & $20 \%$ & $100 \%$ & $100 \%$ \\
\hline $42.85 \%$ & $10 \%$ & $0 \%$ & $0 \%$ \\
\hline $57.14 \%$ & $20 \%$ & $50 \%$ & $50 \%$ \\
\hline $25 \%$ & $0 \%$ & $0 \%$ & $100 \%$ \\
\hline $53.57 \%$ & $10 \%$ & $100 \%$ & $100 \%$ \\
\hline $21.42 \%$ & $40 \%$ & $100 \%$ & $0 \%$ \\
\hline $42.85 \%$ & $0 \%$ & $50 \%$ & $0 \%$ \\
\hline $25 \%$ & $0 \%$ & $0 \%$ & $100 \%$ \\
\hline $25 \%$ & $90 \%$ & $0 \%$ & $100 \%$ \\
\hline $28.57 \%$ & $40 \%$ & $100 \%$ & $50 \%$ \\
\hline & & & \\
\hline
\end{tabular}

Table.6 Comparison of percentage (\%) MDR NFGNB in Various studies

\begin{tabular}{|c|c|}
\hline Study (Year) & \% MDR NFGNB \\
\hline Mathai et al., (2012) & $70 \%$ \\
\hline Jayanthi et al., (2012) & $39.4 \%$ \\
\hline Seema Solanki et al., (2017) & $48.5 \%$ \\
\hline Present study & $\mathbf{4 7 . 6 1 \%}$ \\
\hline
\end{tabular}

*Among 84 Pseudomonas aeruginosa isolates, 3 (3.57\%) isolates showed Pan drug resistance (PDR).

In our study Pseudomonas aeruginosa strains were most susceptible to Colistin $(96.42 \%)$ and least susceptible to Levofloxacin, Ciprofloxacin, Ticarcillin/Clavulanic acid, Tigecycline (25\%) and Minocyclin (21\%). In our study Imipenem resistance was seen in $42.86 \%$ of Pseudomonas aeruginosa strains which is accordance with (Gladstone et al., 2005) study in which it is $42.8 \%$ and in contrast very less, that is $6 \%$ resistance was seen in (Malini et al., 2009) study. In present study Acinetobacter baumanii strains were most susceptible to Colistin (100\%) and Tigecycline $(90 \%)$ but about $76 \%$ of these strains showed high resistance to various drugs similar to (Kirtilaxmi et al., 2014) study. Burkholderia cepacia and Stenotrophomonas maltophilia strains also showed high degree of resistance to various drugs tested. In present study about $47.61 \%$ NFGNB exhibited Multidrug resistance (among all NFGNB, Acinetobacter baumanii, Pseudomonas aeruginosa constituted major percentage of MDRs) and $3.57 \%$ of 
Pseudomonas aeruginosa strains exhibited Pan drug resistance. For this study, Multidrug resistant (MDR) are defined as those isolates resistant to more than three classes of antibiotics, Pan drug resistant (PDR) are those resistant to all the commonly used antibiotics (Minal Vasant Kusalkar et al., 2018). The treatment of infections caused by these organisms is challenging because of high intrinsic and acquired resistance to all commonly used antibiotics (Vikas Gautam et al., 2015). Hence accurate and timely identification and antimicrobial susceptibility testing of NFGNB is necessary for clinicians to choose correct antibiotics for treatment.

In present study, Pseudomonas aeruginosa and Acinetobacter baumanii are most common NFGNB isolated. Correct and rapid identification and antimicrobial susceptibility testing of NFGNB by Vitek 2 is very important because it will help in early initiation of appropriate antimicrobial therapy and proper management of patients. Different species of NFGNB isolated showed varied sensitivity patterns. Emergence and spread of Multidrug resistance and Pan drug resistance strains is an alarming situation and it emphasizes need for improved antibiotic stewardship and infection control measures to be implemented in hospital for better care and management of patients.

\section{References}

Caroline M. O'Hara and J. Michael Miller. 2003. Evaluation of the Vitek 2 IDGNB assay for negative bacilli and comparison with the Vitek GNI_Card. J. Clin. Microbiol., 41(5): 2096-2101.

Deepak Juyal, Rajat Prakash, Shamanth A Shanakarnarayan, Munesh

Sharma, Vikrant Negi and Neelam Sharma. 2013. Prevalence of nonfermenting gram negative and their in vitro susceptibility pattern in a tertiary care hospital of Uttarakhand: A study from foothills of Himalayas. Saudi J. Health Sci., 2(2): 108-112.

Gladstone P, Rajendran P and Brahmadathan KN. 2005. Incidence of carbapenem resistant non fermenting gram negative bacilli from patients with respiratory infections in the intensive care units. Indian J Med Microbiol., 23: 189-91.

Jayanthi S, Jeya, M. 2012. Clinical distribution and antibiotic resistance pattern of non-fermenting gram negative bacilli. Inter J. Phar. Bio Sci., 3(1): 487-494.

Kirtilaxmi K. Benachinmardi, Padmavathy M, Malini J and Naveneeth B. V. 2014. Prevalence of non-fermenting gramnegative bacilli and their in vitro susceptibility pattern at a tertiary care teaching hospital. Journal of the Scientific Society., 41(3): 162-166.

Malini, A, EK Deepa, BN Gokul and SR Prasad. 2009. Non fermenting gramnegative bacilli infections in a tertiary care hospital in Kolar, Karnataka. Journal of Laboratory Physicians., 1(2): 62-66.

Mathai, A.S, Oberoi A, Madhavan S and kaur P. 2012. Acinetobacter infections in a tertiary level intensive care unit in northern India: epidemiology, clinical profiles and outcomes. J. Infect. Public Health., 5(2): 145-152.

Minal Vasant Kusalkar, Agrawal Seema M, and Katkar Vyankatesh J. 2018. A Study of antimicrobial susceptability pattern of clinical isolates of Acinetobacter species at a tertiary care hospital in central India. J. Evolution Med. Dent. Sci., 7(01): 2278-4748.

Providencia Joyanes, Maria del Carmen Conejo, Luis martinnez-Martinez and Evelio J. Perea. 2001. Evaluation of the VITEK 2 system for the identification and susceptibility testing of three species of nonfermenting gram-negative 
rods frequently isolated from clinical samples. J. Clin. Microbiology., 39(9): 3247-3253.

Prudhivi Sumana, Sunita Toleti and Ramesh Babu Myneni. 2017. Prevalence of nonfermenting gram negative bacilli infections and their antimicrobial susceptibility pattern in a tertiary care. International Journal of Current Research., 9(12): 63427-63431.

Ridhima Wadhwa, Yash Sharma, Renuka Pandey Upadhyay and Kumud Bala. 2016. Nosocomial infection by nonfermenting gram negative bacilli in tertiary care hospital: screening and cure. Int J Pharm Pharm Sci., 8(3): 274277.

Seema Solanki, Amisha Sharma and K. Saileela. 2017. Evaluate the distribution of gram negative non fermenting bacteria and their resistant pattern in clinical isolates among the rural population in South India. International Journal of Current Microbiology and Applied Sciences., 6 (5): 461-468.
Shilpa. K. Gokale and S. C. Metgud. 2012. Characterization and antibiotic sensitivity pattern of non-fermenting gram-negative bacilli from various clinical samples in a tertiary care hospital, Belgaum. Journal of pharmaceutical and biomedical sciences., 17(14): 1-5.

Susmitha Simgamsetty, Padmaja Yarlagadda, Bindu Madhav Yenigalla and Ramesh Babu Myneni. 2016. Ease with Vitek 2 systems, Biomerieux in identification of non- lactose fermenting bacteria including their antibiotic drug susceptibility: our experience. Int J Res Med Sci., 4(3): 813-817.

Vikas Gautam, Sunil Kumar, Parvinder Kaur, T. Deepak, Lipika Singhal, Rupinder Tewari and Pallab Ray. 2015. Antimicrobial susceptibility pattern of Burkholderia cepacia complex \& Stenotrophomonas maltophilia over six years (2007-2012). Indian J. Med Res., 142(4): 492-494.

\section{How to cite this article:}

Srividya Yeruva and Vasantha Kabra. 2018. Identification and Antimicrobial Susceptibility testing of Non Fermenting Gram Negative Bacteria by Vitek 2 in a Teaching Hospital in Mahbubnagar. Int.J.Curr.Microbiol.App.Sci. 7(10): 234-240. doi: https://doi.org/10.20546/ijcmas.2018.710.024 